\title{
The dopaminergic system in patients with functional dyspepsia analysed by single photon emission computed tomography (SPECT) and an alpha-methyl-para-tyrosine (AMPT) challenge test
}

\author{
Breg Braak • Jan Booij • Tamira K. Klooker • \\ Rene M. J. van den Wijngaard • Guy E. E. Boeckxstaens
}

Received: 14 July 2011 / Accepted: 22 November 2011 / Published online: 9 December 2011

(C) The Author(s) 2011. This article is published with open access at Springerlink.com

\begin{abstract}
Purpose Functional dyspepsia (FD) is a chronic condition characterized by upper abdominal symptoms without an identifiable cause. While the serotonergic system is thought to play a key role in the regulation of gut physiology, the role of the dopaminergic system, which is important in the regulation of visceral pain and stress, is under-studied. Therefore, this study investigated the dopaminergic system and its relationship with drinking capacity and symptoms in FD patients.

Methods In FD patients and healthy volunteers (HV) the dopaminergic system was investigated by in-vivo assessment of central dopamine D2 receptors (D2Rs) with $\left[{ }^{123} \mathrm{I}\right]$ IBZM SPECT and by an acute, but reversible, dopamine
\end{abstract}

Electronic supplementary material The online version of this article (doi:10.1007/s00259-011-2015-6) contains supplementary material, which is available to authorized users.

B. Braak • T. K. Klooker • G. E. E. Boeckxstaens

Department of Gastroenterology and Hepatology,

Academic Medical Center,

Amsterdam, The Netherlands

\section{J. Booij}

Department of Nuclear Medicine, Academic Medical Center,

Amsterdam, The Netherlands

R. M. J. van den Wijngaard

Tytgat Institute of Liver and Intestinal Research,

Academic Medical Center,

Amsterdam, The Netherlands

G. E. E. Boeckxstaens $(\square)$

Department of Gastroenterology, University Hospital Leuven,

Catholic University Leuven,

Herestraat 45,

Leuven 3000, Belgium

e-mail: Guy.Boeckxstaens@med.kuleuven.be depletion alpha-methyl-para-tyrosine (AMPT) challenge test. A nutrient drink test was performed to investigate the association between maximal ingested volume, evoked symptoms, and D2Rs.

Results The HV subjects comprised 12 women and 8 men (mean age $31 \pm 3$ years), and the FD patients comprised 5 women and 3 men (mean age $39 \pm 5$ years). The FD patients had a lower left plus right average striatal binding potential $\left(\mathrm{BP}_{\mathrm{NP}}\right)$ for the caudate nucleus $(p=0.02)$, but not for putamen $(p=0.15)$, which in the FD patients was correlated with maximal ingested volume $(r=0.756, p=0.03)$. The $\mathrm{D} 2 \mathrm{R}$ $\mathrm{BP}_{\mathrm{NP}}$ in the putamen was correlated with nausea $(r=0.857$, $p=0.01)$. The acute dopamine depletion test, however, failed to reveal differences in prolactin release between the FD patients and the HV subjects.

Conclusion These preliminary data suggest that chronic rather than acute alterations in the dopaminergic system may be involved in the pathogenesis of FD. Further studies are required to reproduce our novel findings and to evaluate to what extent the dopaminergic changes may be secondary to abnormalities in serotonergic pathways.

Keywords Functional dyspepsia $\cdot\left[{ }^{123}\right.$ I] IBZM SPECT . Dopamine D2 receptor · Drink test

\section{Introduction}

Functional dyspepsia (FD) is a chronic condition characterized by upper abdominal symptoms without an identifiable cause. These symptoms include pain, early satiety, fullness, bloating and nausea, and are usually related to food intake [1]. Dyspeptic patients have increased sensitivity to 
distension of the proximal stomach [2] and impaired relaxation of the stomach after food intake [3]. However, the underlying mechanisms giving rise to gastric dysfunction and dyspeptic symptoms remain to be unravelled.

Stress is generally accepted as an important factor triggering or exacerbating dyspeptic symptoms [4]. In rodents, acute stress has been associated with stress-induced visceral analgesia [5], possibly by interfering with the processing of sensory information in the brain. In the brain, pain, pleasure and motivation (emotional cognitive function) are processed in the mesolimbic system, including the ventral tegmental area, nucleus accumbens and anterior cingulate cortex. In particular because these brain areas are rich in dopamine D2 receptors (D2Rs) and activated by the neurotransmitter dopamine [6], dopaminergic pathways are considered important in the regulation of (visceral) pain [7]. Moreover, stress is known to have a detrimental impact on the normal function of the dopaminergic system [8]. Both acute and chronic stress are able to influence levels of dopamine and dopamine receptor function in rodents. However, the association between changes in the dopaminergic system and visceral pain perception has not been investigated.

Another key factor in the regulation of gut physiology, and the interaction with the brain, is the serotonergic (5-HTergic) neurotransmission system. It has been suggested that $5-\mathrm{HT}_{1 \mathrm{~A}}$ receptor hypersensitivity may play a role in $\mathrm{FD}$, which was supported by an increased release of prolactin (PRL) after challenge with buspirone, an agonist of this receptor [9]. However, buspirone is not only an agonist for $5-\mathrm{HT}_{1 \mathrm{~A}}$ receptors, but also a partial antagonist for dopamine D2Rs [10]. Inhibition of D2Rs can stimulate the release of PRL as well. In chronic pain disorders, which are associated with a hypodopaminergic system [11] and are related to FD [12], an increased release of PRL, but not of growth hormone, has been observed after a buspirone challenge test. While the release of growth hormone by buspirone is only mediated by stimulation of the $5-\mathrm{HT}_{1 \mathrm{~A}}$ receptor, is has been suggested that the increased release of PRL is a dopaminergic and not a 5-HT-ergic effect [10]. In addition, in FD, subsequent brain imaging PET studies have shown increased activation of the mesolimbic system (among others in the cingulated cortex) and deactivation of the pregenual anterior cingulate after gastric distension [7, 13]. All these pain-related brain areas are rich in D2Rs and (de)activation of brain areas might correspond to a hypodopaminergic system.

Based on the above, we hypothesized that abnormalities in the central dopaminergic system could be involved in the pathogenesis of FD. We therefore first analysed the in vivo expression of central D2Rs using $\left[{ }^{123} \mathrm{I}\right]$ iodobenzamide ([ $\left.\left[{ }^{123} \mathrm{I}\right] \mathrm{IBZM}\right)$ SPECT in FD patients compared to that in age- and gender-matched healthy volunteers (HV) [14]. In addition, the response of the dopaminergic system to an acute, but reversible, dopamine depletion was investigated using the well-validated alpha-methyl-para-tyrosine (AMPT) challenge test [15]. The advantage of this test is, in contrast to buspirone, that AMPT only interferes with the catecholaminergic system. Finally, on a separate day all participants underwent a nutrient drink test to investigate the correlation between maximal ingested volume, evoked symptoms and the central D2Rs.

\section{Materials and methods}

\section{Study population}

Patients aged 18 to 65 years with a diagnosis of FD as defined by the Rome III criteria [1] were recruited from the gastrointestinal motility unit of the Academic Medical Centre (AMC). Dyspeptic symptoms were scored using the Nepean Dyspepsia Index (NDI) questionnaire [16], a validated method to assess FD symptoms. Based on previous studies, only patients with a NDI score of $>25$ were included $[16,17]$. All patients underwent a careful history, physical examination, upper endoscopy, blood investigation (including thyroid-stimulating hormone, white blood cell count, $\mathrm{C}$ reactive protein) and abdominal ultrasonography. The $\mathrm{HV}$ subjects, recruited by public advertisement, were included if their NDI score was $\leq 5$. Subjects with depression, as diagnosed with a score of $>50$ on the Zung self-rating depression scale [18] and with concomitant diseases, such as diabetes mellitus, epilepsy and cardiovascular disorders, were excluded. Other reasons for exclusion were previous abdominal surgery (except appendectomy and cholecystectomy), gastrooesophageal reflux disease and concurrent use of NSAIDs or antidepressants. Medication likely to interfere with gastrointestinal function or the dopaminergic system (prokinetics) and analgesics were discontinued at least 7 days before entering the study. In all female participants pregnancy was excluded based on a clinical interview and the $\beta$-human chorionic gonadotrophin $(\beta-\mathrm{HCG})$ urine test.

The study protocol was approved by the Medical Ethics Committee of the AMC, Amsterdam. All participants gave their written and informed consent to participate in the study.

\section{Study protocols}

The timeline of the study protocols is shown in Fig. 1.

\section{SPECT imaging: data acquisition}

After ingestion of potassium iodide (three doses of $40 \mathrm{mg}$ on the day before imaging and $80 \mathrm{mg}$ just before imaging) to block thyroid uptake of free radioactive iodide, the selective 
Fig. 1 Study protocol of SPECT imaging and AMPT challenge test in the $8 \mathrm{FD}$ patients and the $20 \mathrm{HV}$ subjects

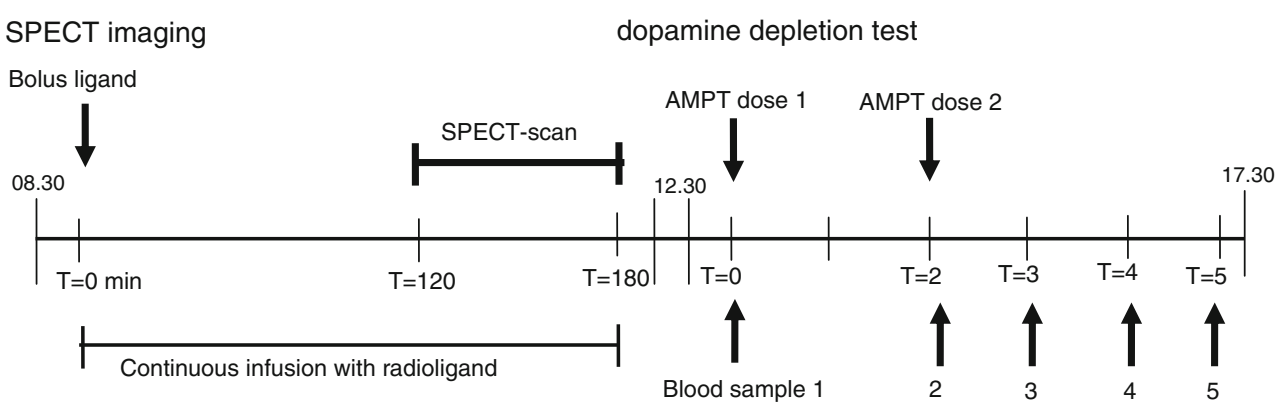

radiolabelled D2R antagonist $\left[{ }^{123} \mathrm{I}\right] \mathrm{IBZM}$ was infused using the sustained equilibrium/constant infusion technique [14, 19]. First a $\left[{ }^{123} \mathrm{I}\right] \mathrm{IBZM}$ dose (specific activity $>200 \mathrm{MBq} /$ nmol and radiochemical purity $>95 \%$ ) of approximately $64 \mathrm{MBq}$ was given as a bolus, followed by a continuous infusion of $48 \mathrm{MBq}$ (dissolved in $0.9 \% \mathrm{NaCl}$ ) for the duration of the experiment (180 min). Acquisition of the images (12-13 slices; $64 \times 64$ matrix) was started $2 \mathrm{~h}$ after the bolus injection. Images were obtained with a 12-detector singleslice brain-dedicated scanner (Neurofocus 810 ) with a fullwidth at half-maximum resolution of approximately $6.5 \mathrm{~mm}$ throughout the 20-cm field of view (http://www.neurophysics. com). After positioning the subject with the head parallel to the orbitomeatal line, axial slices parallel to and upward from the cerebellum to the vertex were acquired in 5-mm steps. The energy window was set at $135-190 \mathrm{keV}$. On the day of the imaging session, the participants were not allowed to smoke or to consume coffee or alcohol to avoid interference with striatal dopamine release $[20,21]$.

\section{SPECT imaging: data processing}

Attenuation correction of all images was performed as described previously [22]. Images were reconstructed in 3-D mode. For quantification, a region-of-interest (ROI) analysis of the two main striatal areas (the caudate nucleus and putamen) was performed, as well as of the occipital cortex (reflecting nonspecific binding). Standard templates (according to the contour of the caudate nucleus, putamen and occipital cortex) with fixed ROIs were manually positioned, without changing size and shape, on the five consecutive axial slices with the highest striatal activity to calculated the mean striatal and mean occipital binding by averaging right and left ROIs. The D2R binding potential $\left(\mathrm{BP}_{\mathrm{NP}}\right.$ [23]) was calculated for each striatal area as the ratio of specific to nonspecific activity (total activity in each striatal area minus total activity in the occipital cortex, divided by total activity in the occipital cortex). $\mathrm{BP}_{\mathrm{NP}}$ for the left and right striatal areas was calculated separately as the ratio of specific to nonspecific activity (activity in left or right striatal areas divided by the occipital activity). In addition, an asymmetry index was calculated to determine differences between the right and left side (activity in the right minus activity in the left striatal area divided by the sum of the right and left activity).

\section{Dopamine depletion by AMPT challenge}

Dopamine depletion was induced by oral administration of AMPT using a fixed total dose of $1,000 \mathrm{mg}$ (four capsules of $250 \mathrm{mg}$ ) $[15,24]$ administered over $2 \mathrm{~h}$, the first dose $(500 \mathrm{mg}$ ) given at noon and the second $2 \mathrm{~h}$ later. Blood samples were collected via an intravenous catheter before and every hour over the $4 \mathrm{~h}$ after AMPT administration. The degree of dopamine inhibition was indirectly measured by PRL using a time-resolved fluoroimmunoassay (DELFIA Prolactin; Wallac Oy, Turku, Finland). The total assay variation ranged from $5.8 \%$ to $7.6 \%$.

\section{Nutrient drink test}

On a separate day and after overnight fasting, participants underwent a nutrient drink test [17] to evaluate the correlation between maximal ingested volume, postprandial symptoms and striatal binding capacity.

Participants were asked to drink $100 \mathrm{ml}$ of Nutridrink (N. V. Nutricia; Zoetermeer, The Netherlands; $1.5 \mathrm{Kcal} / \mathrm{ml}, 39 \%$ fat, $48 \%$ carbohydrates, $13 \%$ protein) every minute until maximal satiation. After each $100 \mathrm{ml}$, symptoms of nausea, bloating, satiety, fullness, burning sensation, pain and hunger were scored on an ordinary symptoms scale ranging from 0 (no sensation) to 5 (severe sensation). When subjects reported a maximum score (5) for one of the symptoms, the corresponding ingested volume was determined as the maximal ingested volume. Thereafter, symptoms were scored every $30 \mathrm{~min}$ on a VAS scale $(0-10 \mathrm{~cm}$; 0 no sensation, 10 worst imaginable) until $3 \mathrm{~h}$ after the test. In addition, the integrated VAS scores for each symptom were calculated by adding each individual symptom (e.g. nausea, pain) during the entire postprandial period, with a maximum score of 70 . The sum of these total scores yielded the total postprandial symptom VAS scores (maximum 490). 
Statistical analysis

Striatal $\mathrm{D} 2 \mathrm{R} \mathrm{BP}_{\mathrm{NP}}$, ingested volume, postprandial symptoms, baseline PRL levels and baseline characteristics including gender and age were compared between groups using the Mann-Whitney $U$-test or the chi-squared test. Spearman's rho correlation coefficients were calculated to investigate the relationship between striatal D2R BP ${ }_{\mathrm{NP}}$, PRL levels, maximal ingested volume and dyspeptic symptoms (correlation coefficient $R \geq 0.7$ was considered relevant). Ingested volumes, postprandial symptoms and PRL levels were compared within groups using the nonparametric Wilcoxon signed ranks test for paired samples. A two-tailed probability value of 0.05 was considered as the significance level. Statistical analyses were performed with SPSS, release 16.0.1 for Windows (SPSS, Chicago, IL).

\section{Results}

\section{Study population}

Eight FD patients and $20 \mathrm{HV}$ subjects participated in the SPECT imaging study. The two groups were comparable and not significantly different regarding age and sex distribution: the FD patients comprised 5 women $(63 \%)$ and 3 men (mean age $39 \pm 5$ years), and the HV subjects comprised 12 women (60\%) and 8 men (mean age $31 \pm 3$ years). In the second part of the study, seven HV subjects declined to participate in the nutrient drink test, dropped out and were not included in the analysis of the drink test.

\section{SPECT imaging}

Compared to the $20 \mathrm{HV}$ subjects, the $8 \mathrm{FD}$ patients had a significantly lower average left plus right striatal $\mathrm{BP}_{\mathrm{NP}}$ for the caudate nucleus $(p=0.02)$, but not for the putamen ( $p=$ 0.15 ; Fig. 2). The asymmetry index (difference between left and right side) was comparable between the FD patients and

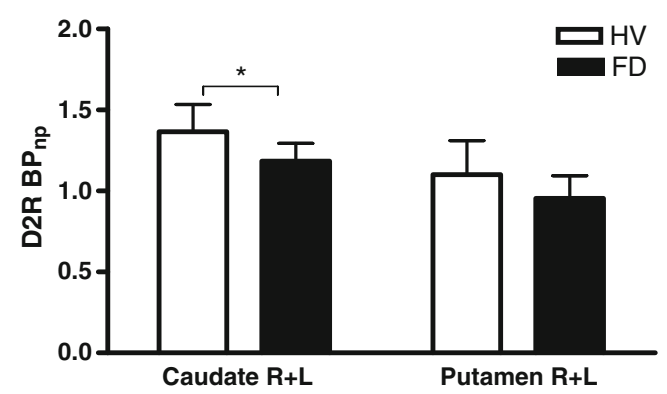

Fig. $2 \mathrm{BP}_{\mathrm{NP}}$ of striatal $\mathrm{D} 2 \mathrm{R}$ (average right plus left, $R+L$ ) in the $8 \mathrm{FD}$ patients and $20 \mathrm{HV}$ subjects in the caudate nucleus and putamen. The data are shown as medians +75 th interquartile ranges $\left(^{*} p=0.02\right.$, Mann-Whitney $U$ test)
HV subjects for the caudate nucleus (FD patients, -0.05 , range -0.1 to 0.1 ; HV subjects, -0.04 , range -0.2 to 0.1 ; NS) and the putamen (FD patients, 0.01 , range -0.1 to 0.1 ; HV subjects, -0.00 , range -0.2 to 0.2 ; NS).

\section{Dopamine depletion by AMPT challenge}

At baseline, the median PRL levels were comparable between the groups (FD patients, $7.5 \mu \mathrm{g} / \mathrm{l}$, range $2.0-12.5 \mu \mathrm{g} /$ 1; HV subjects, $9.0 \mu \mathrm{g} / \mathrm{l}$, range $5.0-17.0 \mu \mathrm{g} / \mathrm{l} ; p=0.06$; Fig. 3a). AMPT challenge significantly increased PRL levels compared to baseline in both groups (Fig. $3 b, p<0.01$ ). The levels then slowly decreased, but were still elevated after $5 \mathrm{~h}$. However, there was no significant difference in PRL levels between the FD patients and HV subjects. The maximum PRL level was also comparable between the groups (FD patients, $86 \mu \mathrm{g} / \mathrm{l}$, range $59-130 \mu \mathrm{g} / \mathrm{l}$; HV subjects, $87 \mu \mathrm{g} / \mathrm{l}$, 30-185 $\mu \mathrm{g} / \mathrm{l} ; \mathrm{NS})$.

\section{Drink test}

In all FD patients and the $13 \mathrm{HV}$ subjects who consented to undergo the nutrient drink test, the maximal ingested volume was comparable (FD patients, $800 \mathrm{ml}$, range 200 1,600 ml; HV subjects, $850 \mathrm{ml}$, range $350-1,500 \mathrm{ml}$; NS). Symptoms including nausea, pain, satiety, fullness and bloating were significantly increased in both groups compared to their baseline values $(p<0.03)$, whereas a burning sensation was only increased in the HV subjects $(p=0.01)$ after the nutrient drink. In the FD patients, nausea was significantly increased compared to the HV subjects directly after the drink test up to $120 \mathrm{~min}$ ( $p<0.04$; Fig. 4). Pain and bloating were also significantly increased in the FD patients compared to the HV subjects at 30 and $120 \mathrm{~min}$, respectively $(p<0.05)$. Other postprandial symptoms were comparable between the groups (Fig. 4 and Supplementary Fig. S1).

In the FD patients, the integrated VAS score (sum of scores at all time points) for nausea was significantly increased $(p<0.02)$ and tended to show an increase for pain $(p<0.06$, Fig. 4 inset) compared to the scores in the HV subjects. Similarly, the total postprandial score was significantly increased in the FD patients compared to the score in the HV subjects (FD patients, 205, range 53-220; HV subjects, 96, range 42-150; $p=0.04)$. Other integrated VAS scores were comparable between the groups (Supplementary Fig. S1). Finally, the maximal ingested volume was negatively correlated with the integrated VAS score for nausea in the HV subjects $(r=-0.681, p=0.02)$, but not in the FD patients $(r=0.296, p=0.5$; see, for example, Supplementary Fig. S2). There was no correlation between maximal ingested volume and the other integrated VAS scores (data not shown). 
Fig. 3 PRL levels in FD patients and HV subjects before and after AMPT challenge. a Individual baseline values (horizontal lines medians; NS, Mann-Whitney $U$ test). b Geometric mean values following AMPT challenge (NS)

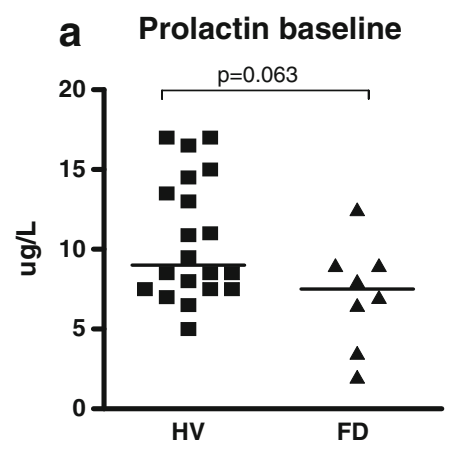

Relationships between striatal D2Rs, maximal ingested volume, postprandial symptoms and PRL levels

As the asymmetry index is comparable between the left and right sides of the brain for each striatal area, correlations were calculated for the average of the two sides. The average $\mathrm{D} 2 \mathrm{R} \mathrm{BP}_{\mathrm{NP}}$ for the caudate nucleus was significantly correlated with maximal ingested volume $(r=0.756 p=0.03)$ in the FD patients, but not in the HV subjects $(r=-0.211, p=$ $0.47)$. The correlation between the average $\mathrm{D} 2 \mathrm{R} \mathrm{BP} \mathrm{NP}_{\mathrm{N}}$ for the putamen and the maximal ingested volume was of borderline
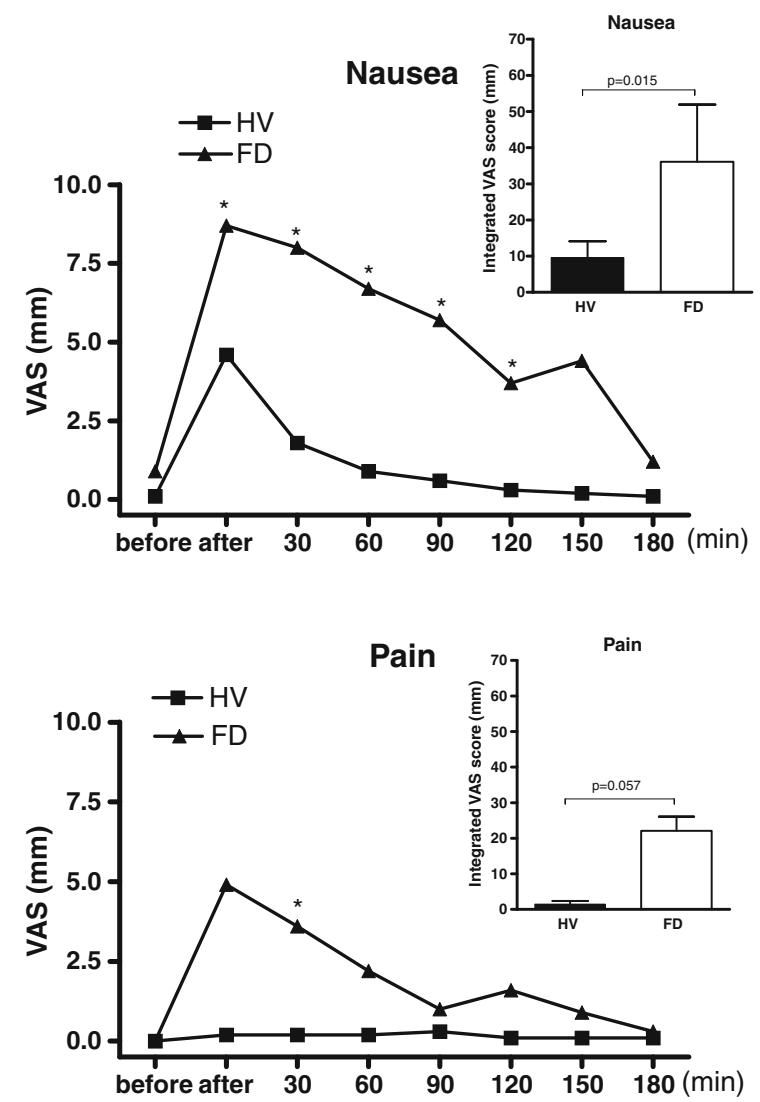

Fig. 4 Postprandial symptoms nausea, pain and bloating in the 8 FD patients and $13 \mathrm{HV}$ subjects. Insets Integrated VAS scores of postprandial symptoms (sum of all VAS scores during the entire postprandial significance in the FD patients $(r=0.683, p=0.06)$, but was not significant in the HV subjects ( $r=-0.178, p=0.54$; Fig. 5).

Regarding evoked symptoms in the FD patients, the average $\mathrm{D} 2 \mathrm{R} \mathrm{BP}_{\mathrm{NP}}$ for the putamen was significantly correlated with the integrated VAS score for nausea $(r=0.857, p=0.01)$, but there was no significant correlation in the HV subjects $(r=$ $0.256, p=0.422$ ). This correlation was not significant in the caudate nucleus (FD patients, $r=0.429, p=0.34$; HV subjects, $r=0.343, p=0.28$; Fig. 6). Other (postprandial) symptoms were not correlated with the average $\mathrm{D} 2 \mathrm{R} \mathrm{BP} \mathrm{NP}_{\mathrm{N}}$ in the striatal areas in either group (data not shown). 
Fig. 5 Relationships between average striatal $\mathrm{D} 2 \mathrm{R} \mathrm{BP} \mathrm{NP}_{\mathrm{N}}$ and maximal ingested volumes (Spearman's rho correlation coefficients)

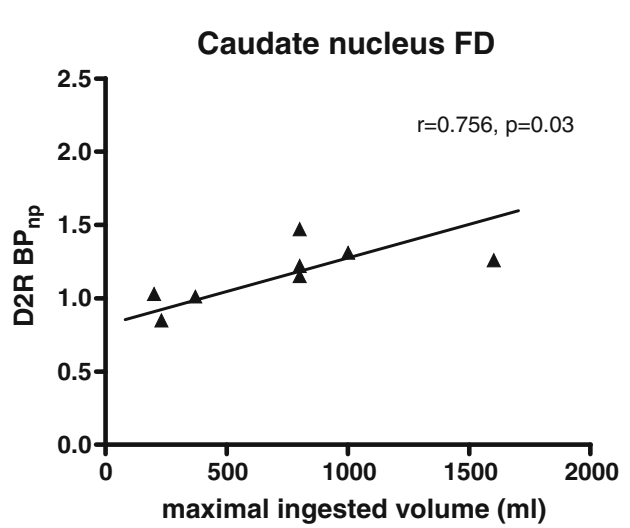

Putamen FD

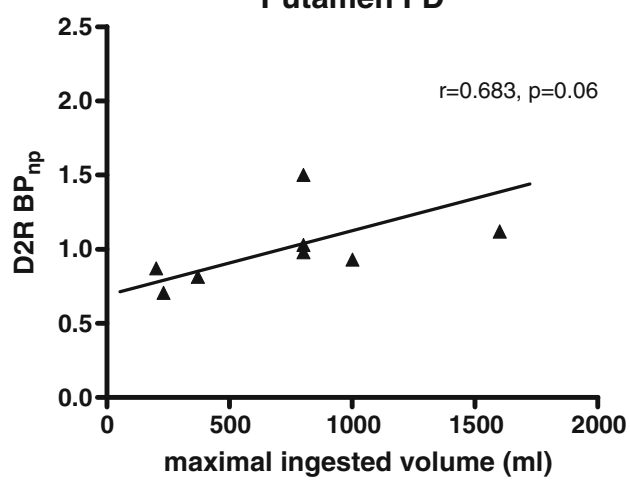

Caudate nucleus HV

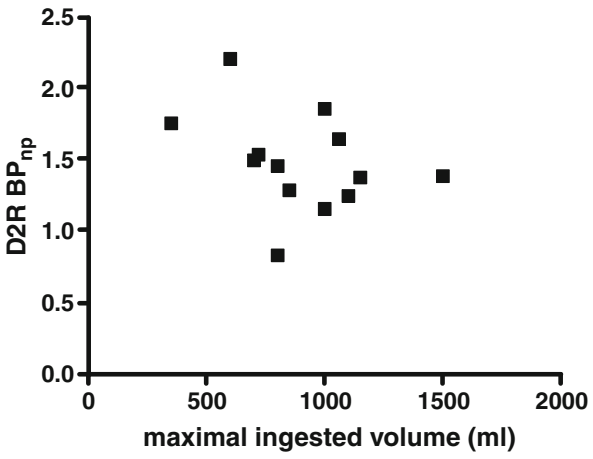

Putamen HV

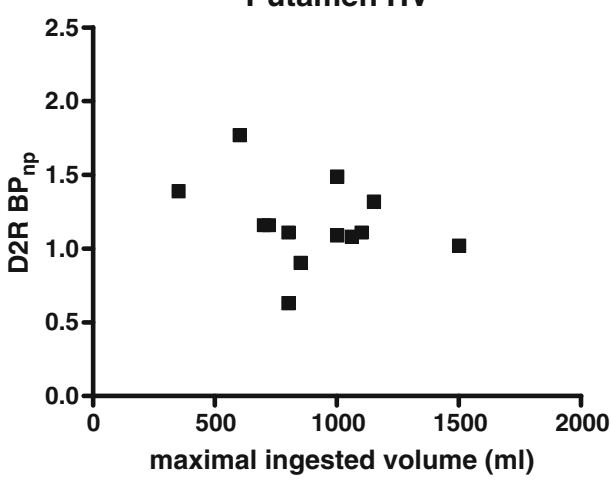

Regarding PRL, there were no significant correlations between the average $\mathrm{D} 2 \mathrm{R} \mathrm{BP}_{\mathrm{NP}}$ in either striatal area or symptoms or maximal ingested volume in either group and baseline PRL or maximum PRL levels. Similarly, there were
Fig. 6 Relationships between average striatal $\mathrm{D} 2 \mathrm{R} \mathrm{BP} \mathrm{NP}_{\mathrm{NP}}$ and integrated VAS scores for nausea (Spearman's rho correlation coefficients)

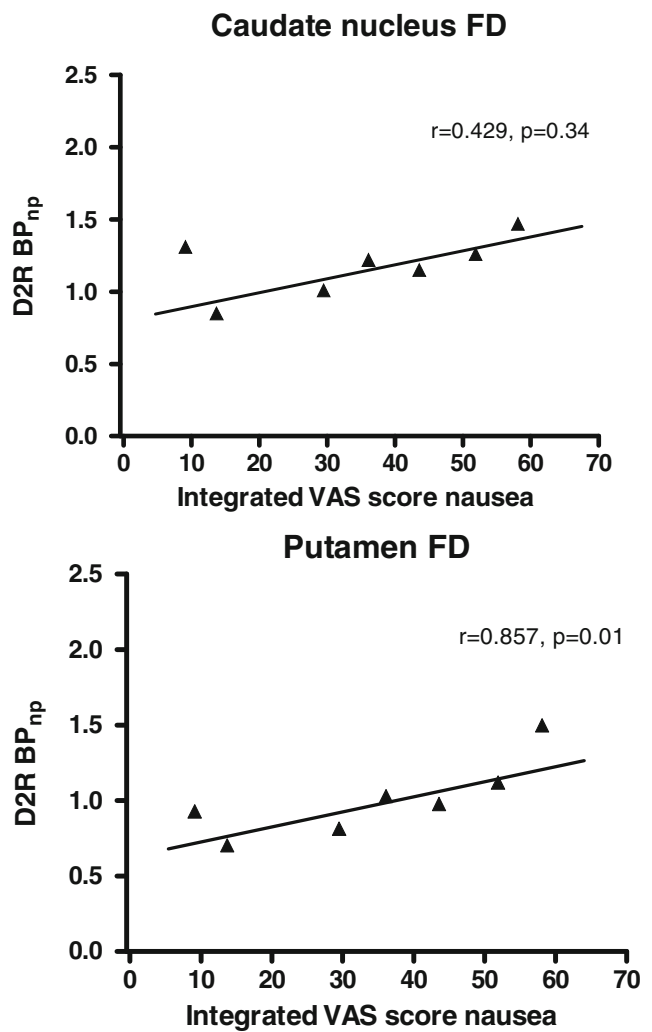

Caudate nucleus HV

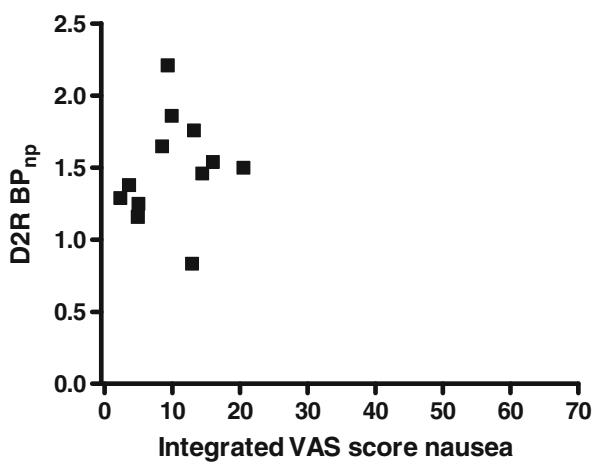

Putamen HV

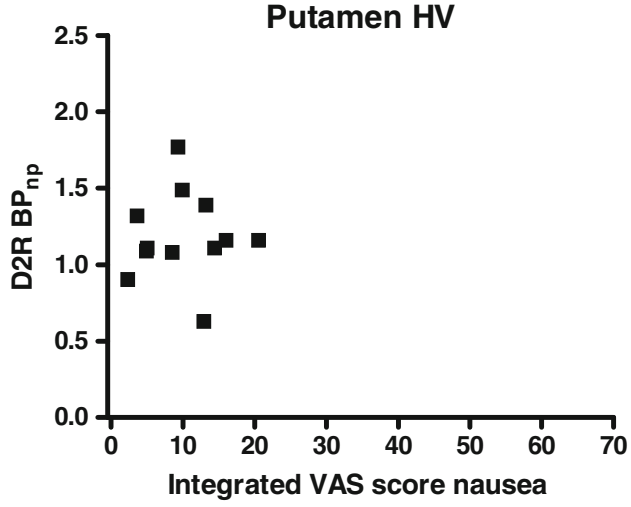


no significant correlations between baseline or maximum PRL levels and postprandial symptoms or maximal ingested volumes (data not shown).

\section{Discussion}

In the present study, we observed a lower $\mathrm{D} 2 \mathrm{R} \mathrm{BP}_{\mathrm{NP}}$ in the caudate nucleus, one of the two main striatal areas, in FD patients, which was significantly correlated with the maximal ingested volume during a drink test. Likewise, the D2R $\mathrm{BP}_{\mathrm{NP}}$ in the putamen was significantly correlated with nausea. After acute dopamine depletion, however, there were no differences in PRL release between the HV subjects and FD patients. These findings suggest that chronic rather than acute alterations in the dopaminergic system may be involved in the pathogenesis of FD. Further studies are, however, required to confirm this hypothesis.

It is widely recognized that stress is an important trigger for functional bowel disorders, including FD [4]. Importantly, stress may alter the dopaminergic system [8] and is particularly associated with lowered D2R binding in the caudate nucleus $[25,26]$. Using PET imaging, the caudate nucleus has been shown to be activated by gastric distension, suggesting involvement of this brain area in visceral perception [7, 13, 27]. In particular because the caudate nucleus is rich in D2Rs, we hypothesized that altered dopaminergic transmission could be involved in the pathogenesis of FD. To this end, we determined the $\mathrm{D} 2 \mathrm{R} \mathrm{BP}_{\mathrm{NP}}$ using SPECT imaging in FD patients and HV subjects. The uptake of radiolabelled D2R antagonist $\left[{ }^{123}\right.$ I]IBZM [14] reflects the proportion of D2Rs not occupied by endogenous dopamine, and is therefore an indirect measure of endogenous dopaminergic tone, i.e. higher levels of endogenous dopamine are associated with a lower binding potential of $\left[{ }^{123} \mathrm{I}\right] \mathrm{IBZM}$, according to the competition model [25].

In this study we showed that in patients with FD, D2R binding in the caudate nucleus was significantly lower than in healthy subjects, together with a tendency to have lower basal PRL levels. Moreover, D2R binding was positively associated with drinking capacity in FD patients. As previous studies have shown reduced drinking capacity in FD patients compared to healthy subjects $[17,28]$, the finding that reduced D2R binding is associated with reduced drinking capacity would further suggest that an increased basal dopaminergic tone may be involved in the pathogenesis of FD. This is compatible with the observation that dopaminergic D2R/D3R antagonists, especially if they pass the blood-brain barrier, for example metoclopramide, effectively reduce nausea, which can be beneficial in FD patients [29]. However, after meal intake (drink test), nausea scores were positively correlated with D2R binding in FD patients. These data would indicate that reduced rather than increased release of dopamine is related to meal-induced nausea. Moreover, reduced levels of dopamine may explain lower $\mathrm{D} 2 \mathrm{R}$ binding as well. For example, the most consistent finding in cocaine addiction is a decrease in striatal D2Rs. An elegant recent study using the AMPT challenge test, however, has shown that this decrease could be attributed to lower rather than higher levels of endogenous dopamine, while the D2R binding capacity did not increase during AMPT depletion [30]. Clearly, different mechanisms are involved in the regulation of dopamine release and symptom generation, but it also has to be emphasized that D2R binding in the basal state may not reflect neurotransmitter release during a physiological process such as food intake. Therefore, further D2R imaging studies assessing D2R availability before and after the use of AMPT are needed to evaluate whether the lower D2R binding in FD patients as observed in the present study could be attributed to higher (or lower) levels of endogenous dopamine.

To further evaluate the hypothesis that dopaminergic transmission in the striatum is altered in FD patients, the effect of acute dopamine depletion by AMPT treatment [15, 24] on PRL release was investigated in both HV subjects and FD patients. As PRL release is inhibited by dopamine, acute dopamine depletion can be used to indirectly assess the dopaminergic tone. As expected, AMPT challenge resulted in a significant increase (almost ten times; Fig. 3) in PRL levels, indicating that the dosage and infusion protocol used were indeed effective. However, no differences in PRL release were detected between FD patients and HV subjects. This finding is in contrast with the reduced $D 2 R$ $\mathrm{BP}_{\mathrm{ND}}$ in the striatal areas observed in $\mathrm{FD}$ patients. One explanation might be that imaging studies reflect the chronic situation, while the AMPT condition reflects a dopaminergic response to an acute dopaminergic intervention. Alternatively, these findings may indicate that the changes in the dopaminergic system are not the primary cause of FD, but may reflect a compensation for changes in another neurotransmitter system, e.g. the 5-HT-ergic system. In the brain, the dopaminergic and 5-HT-ergic systems closely interact. For example, compelling electrophysiological and neurochemical data show that the main 5-HT control of the activity of midbrain dopaminergic neurons is inhibitory $[31,32]$. Therefore, a possible increase in synaptic striatal dopamine levels might not only be induced by increased activity of midbrain dopaminergic neurons per se. A few studies have investigated indirectly the 5-HT-ergic system in FD patients by a buspirone challenge test [9] or a cholecystokinin challenge test [33]. These studies showed an increased perception of visceral sensation after the challenge, probably caused by hypersensitive 5-HT receptors, in particular the 5-HT1 receptor. Stimulation of the 5-HT1 receptor negatively affects the synthesis and release of endogenous 5-HT [34]. Consequently, hypersensitivity of the 5-HT1 
receptor may induce decreased production of 5-HT leading to higher dopamine levels [34]. Interestingly, (acute) tryptophan depletion leads to increased visceral sensitivity in patients with irritable bowel syndrome and in controls $[35,36]$, indicating an important role for reduced 5-HT-ergic transmission in irritable bowel syndrome. Therefore, we cannot exclude the possibility that our present observation of lowered striatal D2R binding induced by altered levels of dopamine may be caused by a hyposerotonergic system, and may rather reflect a compensatory mechanism.

The following limitations of the study should be mentioned. Firstly, the number of patients studied was rather low leading to the risk of a type II error, and therefore our findings need to be confirmed. Moreover the low numbers of patients might explain the discrepancy between the putamen and caudate nucleus. Secondly, striatal D2R binding was assessed using SPECT. Novel high-affinity PET tracers for dopamine D2Rs are now available making the measurement of extrastriatal D2Rs also feasible. Given the role of the anterior cingulate cortex and the mid-cingulated cortex in pain induced by rectal distension [37] and gastric distension [7, 38], it would be of great interest to examine dopamine D2R binding in these brain areas. Furthermore, the spatial resolution of SPECT is lower than that of PET, although we used a braindedicated system with a high spatial resolution. However, IBZM SPECT is a well-validated reproducible method for measuring D2Rs in the striatum [19, 22, 39]. Finally, a strength of our study is that we used a bolus/infusion technique to assess in vivo dopamine D2R binding [14]. This approach is not sensitive to changes in cerebral blood flow, and measures D2Rs in a state of equilibrium.

In conclusion, we are the first to show decreased striatal $\mathrm{D} 2 \mathrm{R}$ binding in the caudate nucleus in FD patients. D2R binding capacity in FD patients was significantly correlated with drinking capacity and evoked nausea suggesting the involvement of altered, probably increased, dopaminergic transmission in the striatum in the pathophysiology of FD. However, to what extent this may be secondary to abnormalities in serotonergic pathways remains to be investigated. We believe that our findings will initiate further studies on the possible role of alterations of the dopaminergic as well as the serotonergic system in the induction of FD, which may eventually lead to a more individualized treatment of FD.

Acknowledgments The authors are grateful to Berber Binnerts and Ana Maldonado for their help in the collection of data and the logistics concerning the analysis of the drink test and dopamine depletion data.

Financial support/conflicts of interest G.E.B. was funded by a grant by the Flemish government (Odysseus Program, Fonds Wetenschappelijk Onderzoek, grant G.0905.08). This grant had no influence on the writing of the report or on the decision to submit the paper for publication.
Open Access This article is distributed under the terms of the Creative Commons Attribution Noncommercial License which permits any noncommercial use, distribution, and reproduction in any medium, provided the original author(s) and source are credited.

\section{References}

1. Tack J, Talley NJ, Camilleri M, Holtmann G, Hu P, Malagelada JR, et al. Functional gastroduodenal disorders. Gastroenterology. 2006;130:1466-79.

2. Coffin B, Azpiroz F, Guarner F, Malagelada JR. Selective gastric hypersensitivity and reflex hyporeactivity in functional dyspepsia. Gastroenterology. 1994;107:1345-51.

3. Berstad A, Hauksen T, Gilja OH, Hveem K, Undeland KA, Wilhelmsen I, et al. Gastric accommodation in functional dyspepsia. Scand J Gastroenterol. 1997;32:193-7.

4. Van Oudenhove L, Vandenberghe J, Geeraerts B, Vos R, Persoons P, Fischler B, et al. Determinants of symptoms in functional dyspepsia: gastric sensorimotor function, psychosocial factors or somatisation? Gut. 2008;57:1666-73.

5. Bradesi S, Schwetz I, Ennes HS, Lamy CM, Ohning G, Fanselow $\mathrm{M}$, et al. Repeated exposure to water avoidance stress in rats: a new model for sustained visceral hyperalgesia. Am J Physiol Gastrointest Liver Physiol. 2005;289:G42-53.

6. Hurd YL, Suzuki M, Sedvall GC. D1 and D2 dopamine receptor mRNA expression in whole hemisphere sections of the human brain. J Chem Neuroanat. 2001;22:127-37.

7. Van Oudenhove L, Vandenberghe J, Dupont P, Geeraerts B, Vos R, Dirix $S$, et al. Abnormal regional brain activity during rest and (anticipated) gastric distension in functional dyspepsia and the role of anxiety: a H(2)(15)O-PET study. Am J Gastroenterol. 2010;105:913-24.

8. Pani L, Porcella A, Gessa GL. The role of stress in the pathophysiology of the dopaminergic system. Mol Psychiatry. 2000;5:14-21.

9. Dinan TG, Mahmud N, Rathore O, Thakore J, Scott LV, Carr E, et al. A double-blind placebo-controlled study of buspirone-stimulated prolactin release in non-ulcer dyspepsia - are central serotoninergic responses enhanced? Aliment Pharmacol Ther. 2001;15:1613-8.

10. Malt EA, Olafsson S, Aakvaag A, Lund A, Ursin H. Altered dopamine D2 receptor function in fibromyalgia patients: a neuroendocrine study with buspirone in women with fibromyalgia compared to female population based controls. J Affect Disord. 2003;75:77-82.

11. Wood PB, Patterson JC, Sunderland JJ, Tainter KH, Glabus MF, Lilien DL. Reduced presynaptic dopamine activity in fibromyalgia syndrome demonstrated with positron emission tomography: a pilot study. J Pain. 2007;8:51-8.

12. Malt EA, Ursin H. Mutilation anxiety differs among females with fibromyalgia and functional dyspepsia and population controls. J Psychosom Res. 2003;54:523-31.

13. Van Oudenhove L, Vandenberghe J, Dupont P, Geeraerts B, Vos R, Dirix $\mathrm{S}$, et al. Regional brain activity in functional dyspepsia: a $\mathrm{H}$ (2)(15)O-PET study on the role of gastric sensitivity and abuse history. Gastroenterology. 2010;139:36-47.

14. Booij J, Korn P, Linszen DH, van Royen EA. Assessment of endogenous dopamine release by methylphenidate challenge using iodine-123 iodobenzamide single-photon emission tomography. Eur J Nucl Med. 1997;24:674-7.

15. Boot E, Booij J, Hasler G, Zinkstok JR, de Haan L, Linszen DH, et al. AMPT-induced monoamine depletion in humans: evaluation of two alternative [123I]IBZM SPECT procedures. Eur J Nucl Med Mol Imaging. 2008;35:1350-6. 
16. Talley NJ, Haque M, Wyeth JW, Stace NH, Tytgat GN, Stanghellini V, et al. Development of a new dyspepsia impact scale: the Nepean Dyspepsia Index. Aliment Pharmacol Ther. 1999;13:225-35.

17. Boeckxstaens GE, Hirsch DP, van den Elzen BD, Heisterkamp SH, Tytgat GN. Impaired drinking capacity in patients with functional dyspepsia: relationship with proximal stomach function. Gastroenterology. 2001;121:1054-63.

18. Zung WW. A self-rating depression scale. Arch Gen Psychiatry. 1965;12:63-70.

19. Laruelle M, Abi-Dargham A, van Dyck CH, Rosenblatt W, ZeaPonce Y, Zoghbi SS, et al. SPECT imaging of striatal dopamine release after amphetamine challenge. J Nucl Med. 1995;36:1182-90.

20. Kaasinen V, Aalto S, Nagren K, Rinne JO. Dopaminergic effects of caffeine in the human striatum and thalamus. Neuroreport. 2004; 15:281-5.

21. Nevo I, Hamon M. Neurotransmitter and neuromodulatory mechanisms involved in alcohol abuse and alcoholism. Neurochem Int. 1995;26:305-36.

22. Booij J, Tissingh G, Boer GJ, Speelman JD, Stoof JC, Janssen AG, et al. [123I]FP-CIT SPECT shows a pronounced decline of striatal dopamine transporter labelling in early and advanced Parkinson's disease. J Neurol Neurosurg Psychiatry. 1997;62:133-40.

23. Innis RB, Cunningham VJ, Delforge J, Fujita M, Gjedde A, Gunn $\mathrm{RN}$, et al. Consensus nomenclature for in vivo imaging of reversibly binding radioligands. J Cereb Blood Flow Metab. 2007;27:1533-9.

24. Verhoeff NP, Kapur S, Hussey D, Lee M, Christensen B, Papatheodorou $\mathrm{G}$, et al. A simple method to measure baseline occupancy of neostriatal dopamine D2 receptors by dopamine in vivo in healthy subjects. Neuropsychopharmacology. 2001;25:21323.

25. Morgan D, Grant KA, Gage HD, Mach RH, Kaplan JR, Prioleau $\mathrm{O}$, et al. Social dominance in monkeys: dopamine D2 receptors and cocaine self-administration. Nat Neurosci. 2002;5:169-74.

26. Laruelle M, D'Souza CD, Baldwin RM, Abi-Dargham A, Kanes SJ, Fingado CL, et al. Imaging D2 receptor occupancy by endogenous dopamine in humans. Neuropsychopharmacology. 1997;17:162-74.

27. Ladabaum U, Minoshima S, Hasler WL, Cross D, Chey WD, Owyang C. Gastric distention correlates with activation of multiple cortical and subcortical regions. Gastroenterology. 2001;120:36976.
28. Tack J, Caenepeel P, Piessevaux H, Cuomo R, Janssens J. Assessment of meal induced gastric accommodation by a satiety drinking test in health and in severe functional dyspepsia. Gut. 2003;52:1271-7.

29. Hiyama T, Yoshihara M, Matsuo K, Kusunoki H, Kamada T, Ito $\mathrm{M}$, et al. Meta-analysis of the effects of prokinetic agents in patients with functional dyspepsia. J Gastroenterol Hepatol. 2007;22:304-10.

30. Martinez D, Greene K, Broft A, Kumar D, Liu F, Narendran R, et al. Lower level of endogenous dopamine in patients with cocaine dependence: findings from PET imaging of $D(2) / D(3)$ receptors following acute dopamine depletion. Am J Psychiatry. 2009;166:1170-7.

31. Di Giovanni G, Esposito E, Di Matteo V. Role of serotonin in central dopamine dysfunction. CNS Neurosci Ther. 2010;16:179-94.

32. Navailles S, De Deurwaerdere P. Presynaptic control of serotonin on striatal dopamine function. Psychopharmacology (Berl). 2011;213:213-42.

33. Chua AS, Keeling PW, Dinan TG. Role of cholecystokinin and central serotonergic receptors in functional dyspepsia. World $\mathrm{J}$ Gastroenterol. 2006;12:1329-35.

34. Frey BN, Rosa-Neto P, Lubarsky S, Diksic M. Correlation between serotonin synthesis and 5-HT1A receptor binding in the living human brain: a combined alpha-[11C]MT and [18F]MPPF positron emission tomography study. Neuroimage. 2008;42:850-7.

35. Clarke G, Fitzgerald P, Cryan JF, Cassidy EM, Quigley EM, Dinan TG. Tryptophan degradation in irritable bowel syndrome: evidence of indoleamine 2,3-dioxygenase activation in a male cohort. BMC Gastroenterol. 2009;9:6.

36. Kilkens TO, Honig A, van Nieuwenhoven MA, Riedel WJ, Brummer RJ. Acute tryptophan depletion affects brain-gut responses in irritable bowel syndrome patients and controls. Gut. 2004;53:1794-800.

37. Morgan V, Pickens D, Gautam S, Kessler R, Mertz H. Amitriptyline reduces rectal pain related activation of the anterior cingulate cortex in patients with irritable bowel syndrome. Gut. 2005;54:601-7.

38. Vandenbergh J, Dupont P, Fischler B, Bormans G, Persoons P, Janssens $\mathrm{J}$, et al. Regional brain activation during proximal stomach distention in humans: a positron emission tomography study. Gastroenterology. 2005;128:564-73.

39. Beukers RJ, Booij J, Weisscher N, Zijlstra F, van Amelsvoort TA, Tijssen MA. Reduced striatal D2 receptor binding in myoclonusdystonia. Eur J Nucl Med Mol Imaging. 2009;36:269-74. 